\title{
Discovery and replication of cerebral blood flow differences in major depressive disorder
}

\author{
Crystal M. Cooper ${ }^{1}$ Cherise R. Chin Fatt ${ }^{1}$ - Peiying Liu ${ }^{2}$ Bruce D. Grannemann ${ }^{1}{ }^{1} \cdot$ Thomas Carmody $^{1}$. \\ Jorge R. C. Almeida ${ }^{3}$ - Thilo Deckersbach ${ }^{4}$ - Maurizio Fava ${ }^{4}$ Benji T. Kurian ${ }^{1}$ - Ashley L. Malchow $\mathbb{D}^{1}$. \\ Patrick J. McGrath ${ }^{5}$. Melvin Mclnnis $\mathbb{1}^{6} \cdot$ Maria A. Oquendo ${ }^{7} \cdot$ Ramin V. Parsey $^{8} \cdot$ Elizabeth Bartlett $^{5}$.

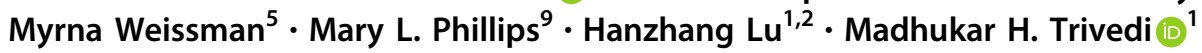

Received: 16 October 2017 / Revised: 6 March 2019 / Accepted: 26 March 2019 / Published online: 6 August 2019

(c) The Author(s), under exclusive licence to Springer Nature Limited 2019

\begin{abstract}
Major depressive disorder (MDD) is a serious, heterogeneous disorder accompanied by brain-related changes, many of which are still to be discovered or refined. Arterial spin labeling (ASL) is a neuroimaging technique used to measure cerebral blood flow (CBF; perfusion) to understand brain function and detect differences among groups. CBF differences have been detected in MDD, and may reveal biosignatures of disease-state. The current work aimed to discover and replicate differences in CBF between MDD participants and healthy controls (HC) as part of the EMBARC study. Participants underwent neuroimaging at baseline, prior to starting study medication, to investigate biosignatures in MDD. Relative CBF (rCBF) was calculated and compared between 106 MDD and 36 HC EMBARC participants (whole-brain Discovery); and 58 MDD EMBARC participants and 58 HC from the DLBS study (region-of-interest Replication). Both analyses revealed reduced $\mathrm{rCBF}$ in the right parahippocampus, thalamus, fusiform and middle temporal gyri, as well as the left and right insula, for those with MDD relative to HC. Both samples also revealed increased rCBF in MDD relative to HC in both the left and right inferior parietal lobule, including the supramarginal and angular gyri. Cingulate and prefrontal regions did not fully replicate. Lastly, significant associations were detected between rCBF in replicated regions and clinical measures of MDD chronicity. These results (1) provide reliable evidence for ASL in detecting differences in perfusion for multiple brain regions thought to be important in MDD, and (2) highlight the potential role of using perfusion as a biosignature of MDD.
\end{abstract}

\section{Introduction}

Major depressive disorder (MDD) is a common, serious, and heterogeneous mood disorder. Despite the conception that it is associated with brain-related changes, brain-based biomarkers have not been established for either use in

Madhukar H. Trivedi

Madhukar.Trivedi@utsouthwestern.edu

1 Department of Psychiatry, University of Texas Southwestern Medical Center, Dallas, TX, USA

2 Department of Radiology, Johns Hopkins University, Baltimore, MD, USA

3 Department of Psychiatry, Dell Medical School, University of Texas Austin, Austin, TX, USA

4 Department of Psychiatry, Massachusetts General Hospital, Boston, MA, USA diagnosis or treatment decision-making. Neuroimaging is continuing to be an increasingly important tool to identify biomarkers for several neurological conditions, including stroke, multiple sclerosis, and Alzheimer's disease, but such biomarkers still require more investigation for use in mood disorders. Magnetic resonance imaging (MRI) provides a
5 Department of Psychiatry, Columbia University, New York State Psychiatric Institute, New York, NY, USA

6 Department of Psychiatry, University of Michigan School of Medicine, Ann Arbor, MI, USA

7 Department of Psychiatry, Perelman School of Medicine, University of Pennsylvania, Philadelphia, PA, USA

8 Department of Psychiatry, Stony Brook University, Stony Brook, NY, USA

9 Department of Psychiatry, University of Pittsburgh School of Medicine, Pittsburgh, PA, USA 
noninvasive medical test that may identify the key brain biosignatures useful for MDD diagnostics, treatment matching, and tracking treatment response [1]. However, there remains a need to replicate and validate the reliability of these tests for MDD before investigating such biosignatures as biomarkers to use clinically.

Arterial spin labeling (ASL) is a noninvasive neuroimaging technique used to measure brain function, similar to blood-oxygen-level-dependent (BOLD) and positron emission tomography (PET). However, opposed to using a radioligand like PET, ASL uses a magnetic pulse to label blood as it perfuses through the brain to provide oxygen and nutrients to tissue. As opposed to measuring connectivity between regions at rest, ASL at rest provides a quantifiable measure of blood perfusion within a given region. Therefore, ASL-derived cerebral blood flow (CBF; perfusion) may be useful to understand brain function in MDD [2]. Yet, only one small study ( $n=44 ; 22$ MDD and 22 healthy controls; HC) has reported absolute CBF differences in the default-mode (i.e., resting/idle state) network before controlling for whole-brain gray matter and age [3]. Another small ASL study was able to accurately distinguish unipolar $(n=18)$ from bipolar depression $(n=18)$ based on differences in $\mathrm{CBF}$ in the anterior cingulate cortex with $81 \%$ accuracy, but did not accurately distinguish MDD from HC [4]. A third study observed lower CBF in MDD $(n=27)$ participants relative to $\mathrm{HC}(n=43)$ within prefrontal and cingulate regions [5]. However, MDD participants were currently being treated for their depression, some of which were classified as non-remitters, which may limit both replicability and generalizability of the findings. Further studies using larger samples are needed to determine if ASL can serve as a clinical tool for MDD diagnosis and treatment decision-making.

The current study, Establishing Moderators/Biosignatures of Antidepressant Response in Clinical Care (EMBARC [6]) is a double-blind, randomized, placebo-controlled trial aiming to detect biosignatures and a treatment response index for MDD [6]. A crucial step toward establishing reliable biomarkers for clinical care is to identify and replicate features (biosignatures) that are altered in the disease-state for the measure of interest. CBF was evaluated as a potential biosignature of MDD using a Discovery-Replication approach. We predicted that participants with MDD would show CBF differences in brain regions involved in major functional networks, including default-mode, emotion, reward, and salience processing (e.g., prefrontal, insula, and cingulate cortices), and they would replicate. Other neuroimaging modalities have observed these networks to be disrupted in MDD (for a review on multi-modal imaging and these pathophysiologic processes in MDD, see [1]). Moreover, we predicted MDD participants would have lower $\mathrm{CBF}$ in the cingulate cortex, inferior and middle frontal gyri, prefrontal cortex, and thalamus, relative to $\mathrm{HC}$, replicating prior ASL work in MDD [5, 7, 8]. The addition of CBF-based findings may aid in identifying brain-based biomarkers of MDD.

To better characterize differences identified in MDD using brain imaging, clinical and translational research often investigate how clinical features relate to these differences. For CBF, one study showed MDD severity was associated with lower perfusion in the cingulate cortex and inferior parietal lobule [3]. So, tertiary to the Discovery-Replication predictions, we hypothesized that clinical features related to MDD chronicity and severity would also associate with perfusion levels, although the directions were exploratory. Characterizing biosignature-clinical relationships may augment diagnostic and treatment response indices.

\section{Materials and methods}

\section{Design and overview}

EMBARC MDD participants were scanned using MRI, including an ASL sequence, pretreatment and one week after starting treatment. All participants' data were collected at one of four sites. Baseline CBF was compared between HC and MDD groups split into Discovery-Replication, 2/3-1/3 subgroups. The Discovery model was exploratory using a whole-brain approach with a less stringent $p$-value threshold of standard cluster size for ASL data. This would allow both corrected and uncorrected significant Discoveryderived regions to be investigated as replicable or spurious upon Replication. The Replication model was conservative using a region of interest (ROI) approach with a more stringent threshold correcting for multiple comparisons. Work following similar designs can be found in GenomeWide Association Study literature [9].

\section{Participants}

EMBARC included 200 MDD participants and $40 \mathrm{HC}$. Additional HC $(n=58)$ came from the Dallas Lifespan Brain Study (DLBS) and were used to augment the EMBARC HC sample and provide independent validation for Discovery-Replication data analysis sets. Participants were 18-65 years old and fluent in English. MDD participants were diagnosed using the Structural Clinical Interview for DSM-IV [10], had early-onset (before age 30), chronic (two or more episodes) MDD, were not undergoing treatment for MDD, antidepressant medication-free for 21 days (5 weeks for fluoxetine) with no failed antidepressant trials of adequate dose and duration (as defined by the MGHATRQ), in the current episode or inadequate response or poor tolerability to study medication. EMBARC HC 
Table 1 Demographic and clinical characteristics for the Discovery and Replication datasets

\begin{tabular}{|c|c|c|c|c|}
\hline & \multicolumn{2}{|l|}{ Discovery dataset } & \multicolumn{2}{|c|}{ Replication dataset } \\
\hline & $\begin{array}{l}\text { EMBARC healthy } \\
\text { controls }\end{array}$ & $\begin{array}{l}\text { EMBARC } \\
\text { MDD sample }\end{array}$ & $\begin{array}{l}\text { DLBS healthy } \\
\text { controls }\end{array}$ & $\begin{array}{l}\text { EMBARC MDD } \\
\text { replication sample }\end{array}$ \\
\hline No. of participants & 36 & 106 & 58 & 58 \\
\hline Age, mean years (SE) & $37.49(2.62)$ & $37.65(1.32)$ & $37.59(1.67)$ & $37.71(1.71)$ \\
\hline $\begin{array}{l}\text { Degree of right-handedness/ } \\
\text { ambidextrous } 1-100 \text {, Mean (SE) }\end{array}$ & $69.56(9.08)$ & $68.53(4.82)$ & Not collected & $73.75(6.31)$ \\
\hline Female $(\%)$ & $24(68.6)$ & $67(63.2)$ & $36(62.1)$ & $36(62.1)$ \\
\hline \multicolumn{5}{|l|}{ Race/ethnicity } \\
\hline Caucasian $(\%)$ & $22(62.9)$ & $7469.8)$ & $49(84.5)$ & $37(63.8)$ \\
\hline Afr. Amer. (\%) & $8(22.9)$ & $22(20.8)$ & $4(6.9)$ & $12(20.7)$ \\
\hline Asian $(\%)$ & $3(8.6)$ & $5(4.7)$ & $2(3.5)$ & $5(8.6)$ \\
\hline Amer. Indian/Alask. Nat. (\%) & $0(0)$ & $1(0.9)$ & $0(0)$ & $0(0)$ \\
\hline Nat. Haw./Pacif. Islander (\%) & $0(0)$ & $0(0)$ & $0(0)$ & $0(0)$ \\
\hline Other $(\%)$ & $2(5.7)$ & $4(3.8)$ & $3(5.2)$ & $4(6.9)$ \\
\hline Years of education, Mean (SE) & $15.76(.79)$ & $14.71(.25)$ & $16.72(.37)$ & $14.72(.35)$ \\
\hline QIDS-SR mean score (SE) & $1.43(.21)$ & $18.56(.29)$ & Not collected & $18.12(.33)$ \\
\hline HAM-D-17 Mean score (SE) & $.54(.13)$ & $18.92(.46)$ & Not collected & $18.74(.57)$ \\
\hline Age of onset (SE) & $0(0)$ & $16.00(.58)$ & Not collected & $17.12(.86)$ \\
\hline Number of MDD episodes (SE) & $0(0)$ & $11.98(2.24)$ & Not collected & $8.72(1.93)$ \\
\hline
\end{tabular}

$M D D$ Major Depressive Disorder, SE standard error, QIDS-SR Quick Inventory of Depressive Symptomatology-Self-Report, HAM-D Hamilton Scale for Depression participants had no history of mental disorders and scored $\leq 8$ on the Quick Inventory of Depressive SymptomatologySelf-Report (QIDS-SR), while MDD participants scored $\geq 14$ [11]. For DLBS inclusion, HC participants had to be predominately right handed, of good medical condition, and cognitively healthy. MDD participants were excluded if any other mental disorder was primary to MDD, if they had a lifetime history of psychotic depressive, schizophrenic, bipolar (I, II, or NOS), schizoaffective, other Axis I psychotic disorders, eating disorder, or had the presence of a medical condition that would compromise MDD-specific findings ${ }^{1}$. Both studies implemented general MRI exclusion criteria. See Table 1 for demographics. The study was approved by the Institutional Review Board at each site. All participants signed informed consent.

\section{Data acquisition and imaging parameters}

EMBARC MRI data were collected at four sites on 3T scanners: Columbia University (CU-General Electric), Massachusetts General Hospital (MG-Siemens), University of Michigan (UM-Philips), and the University of Texas Southwestern Medical Center (TX-Philips). DLBS was

\footnotetext{
${ }^{1}$ Exclusionary medical conditions included the presence of a neurological condition requiring an anticonvulsant; a medical condition not stable with medication, required hospitalization or deemed clinically relevant by the investigators; or any abnormal laboratory results the site principal investigator considered clinically significant.
}

collected at TX. ASL was performed at the end of a sevensequence protocol ${ }^{2}$. ASL was acquired last for two reasons: (1) it was the more exploratory scan of the protocol, and (2) the completion of two non-task scans prior to the ASL sequence (>20 min), would allow return of resting-state function less confounded by residual task function.

All ASL sequences implemented a resting-state, pseudocontinuous technique with a $1516 \mathrm{~ms}$ labeling duration and 1500 post-labeling delay. Other ASL parameters were: $\mathrm{TR} / \mathrm{TE}=4460 \mathrm{~ms} / 17 \mathrm{~ms}$ for EMBARC, $4150 \mathrm{~ms} / 13 \mathrm{~ms}$ for DLBS, in-plane resolution $3.4 \times 3.4 \mathrm{~mm}^{2}$, with $5 \mathrm{~mm}$ thickness covering the whole brain, field of view $(\mathrm{FOV})=$ $220 \times 220 \times 145 \mathrm{~mm}$ for EMBARC, $240 \times 240 \times 135 \mathrm{~mm}$ for DLBS, matrix $=64 \times 64$; flip angle $=90^{\circ}$, multi-slice acquisition in ascending order, 29 slices for EMBARC and 27 slices for DLBS, 70 dynamics, no background suppression, with scan duration of approximately $5 \mathrm{~min}$. These imaging parameters are generally compatible with recommendations from the recent consensus paper [2]. T1-weighted, high-resolution, structural-3D sagittal images were acquired in the same session.

\footnotetext{
${ }^{2}$ Order of sequences (total scan time $\approx 1 \mathrm{~h}$ ): high-resolution structural MRI (MPRAGE $\approx 5 \mathrm{~min}$ ), pre-task resting-state BOLD ( $\approx 6$ min; B0 $\approx 2$ min), event-related BOLD (emotion conflict task $\approx 13$ $\mathrm{min}$ ), event-related BOLD (reward task $\approx 8 \mathrm{~min}$ ), post-task resting-state BOLD ( $\approx 6 \mathrm{~min}$ ), diffusion tensor imaging (DTI $\approx 15 \mathrm{~min}$ with B0), and arterial spin labeling $(\approx 5 \mathrm{~min})$.
} 


\section{Data processing and analysis}

Of the $40 \mathrm{HC}$ and 200 MDD EMBARC participants, $36 \mathrm{HC}$ and 164 MDD baseline ASL scans were used. Others were not collected or excluded due to poor quality (i.e., incomplete sequence, artifact, signal loss, missing brain due to poor FOV placement, and/or excessive motion). All 58 DLBS HC scans were usable. We implemented relative $\mathrm{CBF}(\mathrm{rCBF})$ to detect and replicate differences between $\mathrm{HC}$ and MDD. Each participant's rCBF is the normalization of their absolute $\mathrm{CBF}(\mathrm{aCBF})$, which divides the $\mathrm{aCBF}$ of each voxel by the whole-brain averaged aCBF. CBF is reportedly reliable in $\mathrm{HC}$ populations across scanning sessions (for EMABRC HC data see [12]) [13]. However, rCBF reportedly has greater sensitivity and reliability in detecting differences in perfusion between groups when compared to aCBF [14]. rCBF assists in controlling for individual differences that are not disease-specific (e.g., gender). rCBF's normalization process is also useful to decrease cross-site differences introduced across scanners. Figure 1 depicts $\mathrm{aCBF}$ and $\mathrm{rCBF}$ for this dataset.

The baseline ASL data were preprocessed and analyzed with SPM8 (Wellcome Department of Cognitive Neurology, UK) in Matlab (MathWorks, Natick, MA). Processing followed: (1) realignment of the ASL time-series to correct for head motion, (2) generation of perfusion-weighted image series by pairwise subtraction of the label and control functional images, (3) conversion to aCBF image series based on a single-compartment ASL perfusion model (a center, thalamiclevel slice of the control image was used as a global index representing equilibrium magnetization, i.e. M0), (4)

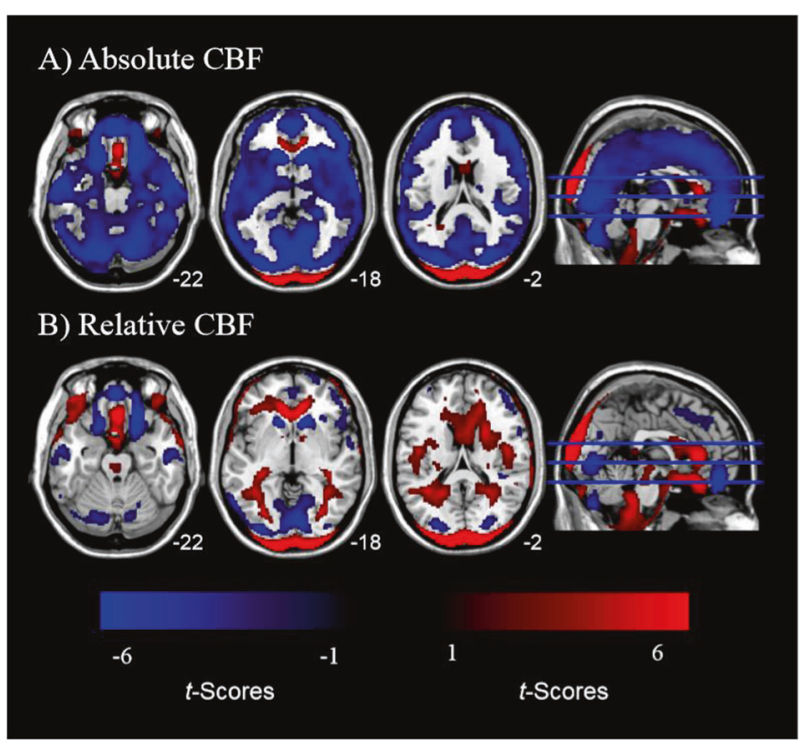

Fig. 1 Site effect for a absolute cerebral blood flow (CBF) and b relative CBF. Regions for which site had a positive effect are in Red. Regions for which site had a negative effect are in Blue generation of a mean CBF image for each participant, (5) co-registration of the mean image with the anatomical image, (6) normalization to the Montreal Neurological Institute (MNI) template, (7) resampling of CBF image to $2 \times 2 \times$ $2 \mathrm{~mm}$ and smoothed with full-width at half-maximum $8 \mathrm{~mm}$ kernel within gray and white matter, and (8) extracting the rCBF image by dividing out the aCBF global individual mean from each aCBF voxel ${ }^{3}$.

Data for rCBF analyses were divided into Discovery and Replication sets modeling precision medicine approaches (i.e., detection in larger to smaller samples). EMBARC HC were used for Discovery, and DLBS age- and gendermatched HC were used for Replication to serve as independent and external validation set (see Fig. 2). MDD participants used for the Discovery and Replication datasets were pseudo-randomly assigned, controlling for important characteristics: site, age, gender, race, handedness, education level, MDD severity, age of MDD onset, and number of episodes (see Table 1).

For Discovery, a whole-brain, analysis of variance (ANOVA) on Group (HC, MDD), controlling for site as a fixed effect (CU, MG, TX, UM), was conducted on rCBF at $p<0.05$ (cluster-level; and 0.01 corrected cluster-level) and extent threshold of 100 continuous voxels using SPM8. The ANOVA compared the 36 EMBARC HC to 106 EMBARC MDD participants. Gray matter coordinates for peak voxels in each significant Discovery cluster, reaching greater than $p=0.001$ voxel significance, were used to build $10 \mathrm{~mm}$ ROI-spheres in MarsBar. ROI-sphere extractions were used for Replication analysis. Fit and significance of ROIspheres were confirmed for the Discovery sample prior to using in Replication sample (all $f>4.10 ; p<0.04$ ). For Replication, Group (HC, MDD) ROI ANOVAs were conducted on rCBF means at $p<0.05$ (Bonferroni adjustment), also controlling for site as a fixed effect using SPSS (IBM, New York, US). Replication ANOVAs compared the 58 DLBS HC to the remaining 58 EMBARC MDD participants. Effect sizes (partial eta-squared; $\eta_{\mathrm{p}}{ }^{2}$ ) for each Replication ROI are reported alongside $p$-values to provide additional information as to the relevance, or promise, of a given region being a biosignature applied to individual cases. Brain regions were defined by the AAL atlas and Brodmann areas using MNI coordinates [15].

In addition to observing disease-state differences in rCBF between $\mathrm{HC}$ and MDD groups, correlations were conducted on those regions that replicated, using the Replication MDD sample, to explore relationships between rCBF and MDD clinical features. Uncorrected Spearman's

\footnotetext{
3 Prior to $\mathrm{rCBF}$ analyses, equivalency of aCBF was confirmed between $\mathrm{HC}$ and MDD groups, $f=0.06 ; p=0.80$, and between TX DLBS HC and TX EMBARC HC as to code them under the TX site, $t=-0.03 ; p=0.98$.
} 


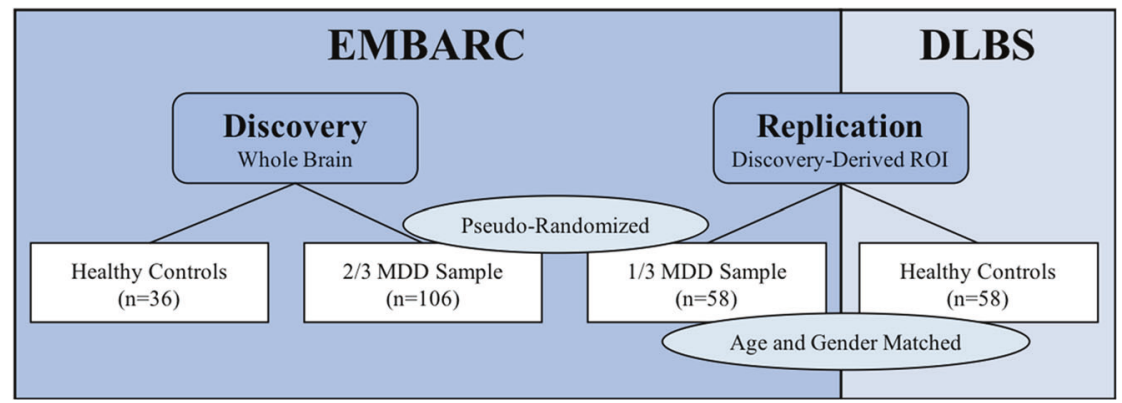

Fig. 2 Study sample and analytic flow diagram. EMBARC participants with major depressive disorder (MDD) were pseudo-randomly divided into 2/3-1/3 Discovery-Replication analyses. EMBARC healthy controls were used for Discovery whole-brain analysis. DLBS healthy controls were used for Replication region of interest (ROI) analyses derived from the Discovery clusters rho correlations were conducted on $\mathrm{rCBF}$ and clinical measures of MDD chronicity, using duration of current episode (months), length of illness (years), age of onset (year of age), and number of major depressive episodes (MDEs), as well as MDD severity, using both the QIDS-SR, self-reported inventory of major symptoms, and the 17-item Hamilton Depression Rating Scale (HAM-D $\left.{ }_{17}\right)$ [16], a standard measure of symptom severity.

\section{Results}

\section{Lower rCBF in MDD than HC}

\section{Discovery sample}

The MDD group showed significantly lower perfusion than HC during Discovery analyses in five clusters: Cluster 1 -a large cluster encompassing bilateral cerebellar and midbrain regions, extending anteriorly to the right thalamus, parahippocampal gyrus, fusiform gyrus, and insula; Cluster 2-the right posterior cingulate cortex; Cluster 3-the left inferior temporal gyrus; Cluster 4-the right middle temporal gyrus and temporal pole; and Cluster 5-the left insula (marginally significant). See Table 2 for detailed cluster information and Fig. 3 for cluster depictions.

\section{Replication sample}

Discovery-derived ROIs that had significantly lower perfusion in the MDD group compared to $\mathrm{HC}$ upon Replication included: Cluster 1 ROIs-the cerebellum, parahippocampal gyrus, fusiform gyrus, and insula, with the thalamus being marginally significant; Cluster 4 ROI-the right middle temporal gyrus and pole (marginally significant); and Cluster 5 ROI-the left insula. See Table 2 for detailed cluster information and effect sizes, and Fig. 3 for cluster depictions.

\section{Greater rCBF in MDD than HC}

\section{Discovery sample}

The MDD group showed significantly greater perfusion than HC during Discovery analyses in five clusters: Cluster 1-the left ventromedial prefrontal cortex extending dorsally to the ventral and dorsal anterior cingulate cortex; Cluster 2-the dorsolateral prefrontal cortex and anterior prefrontal cortices; Cluster 3-the right inferior parietal lobule containing the angular and supramarginal gyri; Cluster 4-left inferior parietal lobule containing the angular and supramarginal gyri; and Cluster 5 -the right precuneus. See Table 2 for detailed cluster information and Fig. 3 for cluster depictions.

\section{Replication sample}

Discovery-derived ROIs that had significantly greater perfusion in the MDD group compared to HC upon Replication included: Cluster 2 ROIs-the left anterior prefrontal and dorsolateral prefrontal cortex; Cluster 3 ROI-the right inferior parietal lobule cluster, including the angular and supramarginal gyri; and Cluster 4 ROI-the left inferior parietal lobule cluster, including the angular and supramarginal gyri. However, the Cluster 2 ROIs were not replicated in the expected direction. HC participants had greater perfusion in these regions than MDD participants. See Table 2 for detailed cluster information and effect sizes, and Fig. 3 for cluster depictions.

\section{Exploratory correlations between $\mathrm{rCBF}$ and clinical features}

For replicated ROIs that had significantly lower $\mathrm{rCBF}$ in the MDD group relative to $\mathrm{HC}$, right parahippocampal gyrus rCBF was positively correlated with duration of current episode, such that longer duration was associated with higher perfusion $\left(r_{\mathrm{s}}=0.26 ; p<0.05\right)$. However, the right 
Table 2 Clusters, i.e., brain regions, in which the healthy control (HC) and major depressive disorder (MDD) groups showed differences in relative cerebral blood flow (rCBF; perfusion) for Discovery and Replication analyses separated by contrast, regions in which those with MDD had lower perfusion and regions in which they had greater perfusion relative to $\mathrm{HC}$

\begin{tabular}{|c|c|c|c|c|c|c|c|c|}
\hline $\begin{array}{l}\text { Discovery cluster } \\
\text { Inset replication region of interest }\end{array}$ & $\begin{array}{l}\text { MNI coordinates } \\
(\mathrm{x}, \mathrm{y}, \mathrm{z})\end{array}$ & BA & $\begin{array}{l}\text { No. } \\
\text { of voxels }\end{array}$ & $\begin{array}{l}t \text {-value } \\
f \text {-value }\end{array}$ & $p$-value & $\eta_{\mathrm{p}}^{2}$ & $M$ & SD \\
\hline \multicolumn{9}{|l|}{$\mathrm{MDD}<\mathrm{HC}$} \\
\hline Cluster 1-Red & $20,-26,-22$ & & 9617 & 5.59 & $0.001^{b}$ & & & \\
\hline Right cerebellum & $20,-26,-22$ & & & 6.02 & 0.02 & 0.05 & 1.23 & 0.24 \\
\hline Right cerebellum & $14,-30,-16$ & & & 2.00 & 0.16 & 0.02 & 1.33 & 0.28 \\
\hline Right thalamus & $20,-16,2$ & & & 3.48 & 0.06 & 0.03 & 0.89 & 0.16 \\
\hline Right parahippocampus & $28,-12,-28$ & 27 & & 32.63 & 0.001 & 0.23 & 0.90 & 0.20 \\
\hline Right fusiform gyrus & $32,-42,-14$ & 37 & & 12.92 & 0.001 & 0.10 & 1.14 & 0.17 \\
\hline Right insula, anterior & $40,-4,-4$ & 13 & & 18.46 & 0.001 & 0.14 & 1.30 & 0.24 \\
\hline Cluster 2-Blue & $4,-34,26$ & & 1078 & 3.90 & $0.04^{b}$ & & & \\
\hline Right posterior cingulate, ventral & $5,-42,27$ & 23 & & 2.26 & 0.14 & 0.02 & 1.54 & 0.25 \\
\hline Cluster 3-Green & $-62,-46,-22$ & & 1037 & 4.45 & $0.05^{b}$ & & & \\
\hline Left inferior temporal gyrus & $-62,-46,-22$ & 20 & & 0.47 & 0.50 & 0.01 & 0.58 & 0.15 \\
\hline Left inferior temporal gyrus & $-56,-52,-20$ & 20 & & 2.93 & 0.09 & 0.03 & 0.84 & 0.18 \\
\hline Cluster 4-Yellow & $58,16,-30$ & & 304 & 3.33 & $0.05^{\mathrm{a}}$ & & & \\
\hline Right middle temporal gyus, pole & $58,16,-30$ & 38 & & 3.50 & 0.06 & 0.03 & 0.55 & 0.17 \\
\hline Right middle temporal gyus & $68,-4,-22$ & 21 & & 2.38 & 0.13 & 0.02 & 0.56 & 0.13 \\
\hline Cluster 5-Violet & $-42,10,-10$ & & 243 & 3.71 & $0.07^{\mathrm{a}}$ & & & \\
\hline Left insula & $-42,10,-10$ & 13 & & 6.71 & 0.01 & 0.06 & 1.38 & 0.27 \\
\hline \multicolumn{9}{|l|}{$\mathrm{MDD}>\mathrm{HC}$} \\
\hline Cluster 1-Red & $-8,28,-16$ & & 445 & 3.85 & $0.02^{\mathrm{a}}$ & & & \\
\hline Left ventromedial prefrontal cortex & $-8,28,-16$ & 11 & & 0.46 & 0.50 & 0.004 & 0.61 & 0.18 \\
\hline Left anterior cingulate, dorsal & $-8,38,12$ & 32 & & 0.01 & 0.96 & 0.001 & 0.91 & 0.19 \\
\hline Cluster 2-Blue & $-10,56,12$ & & 318 & 3.34 & $0.05^{\mathrm{a}}$ & & & \\
\hline $\begin{array}{l}\text { Left superior medial frontal gyrus, } \\
\text { anterior prefrontal }\end{array}$ & $-10,56,12$ & 10 & & 6.62 & 0.01 & 0.06 & 0.98 & 0.15 \\
\hline $\begin{array}{l}\text { Left middle frontal gyrus, dorsolateral } \\
\text { prefrontal }\end{array}$ & $-30,44,20$ & 46 & & 7.14 & 0.01 & 0.06 & 1.12 & 0.17 \\
\hline Cluster 3-Green & $58,-46,44$ & & 638 & 3.81 & $0.01^{\mathrm{a}}$ & & & \\
\hline $\begin{array}{l}\text { Right inferior parietal lobule, } \\
\text { supramarginal gyrus }\end{array}$ & $58,-46,44$ & 40 & & 47.48 & 0.05 & 0.30 & 0.97 & 0.18 \\
\hline $\begin{array}{l}\text { Right inferior parietal lobule, } \\
\text { angular gyrus }\end{array}$ & $46,-68,40$ & 39 & & 36.28 & 0.001 & 0.25 & 1.01 & 0.18 \\
\hline Cluster 4-Yellow & $-60,-42,42$ & & 372 & 2.84 & $0.03^{\mathrm{a}}$ & & & \\
\hline $\begin{array}{l}\text { Left inferior parietal lobule, } \\
\text { supramarginal gyrus }\end{array}$ & $-60,-42,42$ & 40 & & 37.73 & 0.001 & 0.25 & 0.86 & 0.20 \\
\hline $\begin{array}{l}\text { Left inferior parietal lobule, } \\
\text { angular gyrus }\end{array}$ & $-44,-68,44$ & 39 & & 58.87 & 0.001 & 0.35 & 0.94 & 0.21 \\
\hline Cluster 5-Violet & $4,-60,38$ & & 284 & 3.70 & $0.05^{\mathrm{a}}$ & & & \\
\hline Right precuneus & $4,-60,38$ & & & 0.007 & 0.93 & 0.001 & 1.50 & 0.21 \\
\hline
\end{tabular}

Whole-brain clusters from the Discovery analysis are represented in bold

Regions of interest used in the Replication analysis are presented below their corresponding Discovery cluster in italics

$M N I$ Montreal Neurological Institute, $B A$ Brodmann Area, $M$ means, $S D$ standard deviations for replication cluster data

${ }^{\mathrm{a} U n c o r r e c t e d ~ c l u s t e r-l e v e l ~}$

${ }^{\mathrm{b}}$ Corrected cluster-level

anterior insula negatively correlated with age of onset $\left(r_{\mathrm{s}}=\right.$ $-0.26 ; p=0.05)$, such that younger age of onset was associated with higher perfusion, while the left insula negatively correlated with length of illness $\left(r_{\mathrm{s}}=-0.29 ; p<0.03\right)$, such that longer length of illness was associated with lower perfusion. Despite only reaching marginal significance upon replication, perfusion in the thalamus positively correlated with length of illness $\left(r_{\mathrm{s}}=0.46 ; p<0.001\right)$ and number of MDEs $\left(r_{\mathrm{s}}=0.32 ; p<0.02\right)$, such that longer length of illness and higher number of MDEs was associated with higher perfusion. Only perfusion in the middle temporal pole positively correlated with depression severity (QIDS-SR), such that higher depression severity was associated with higher perfusion $\left(r_{\mathrm{s}}=\right.$ $0.28 ; p=0.03$ ). For regions that had significantly higher $\mathrm{rCBF}$ 


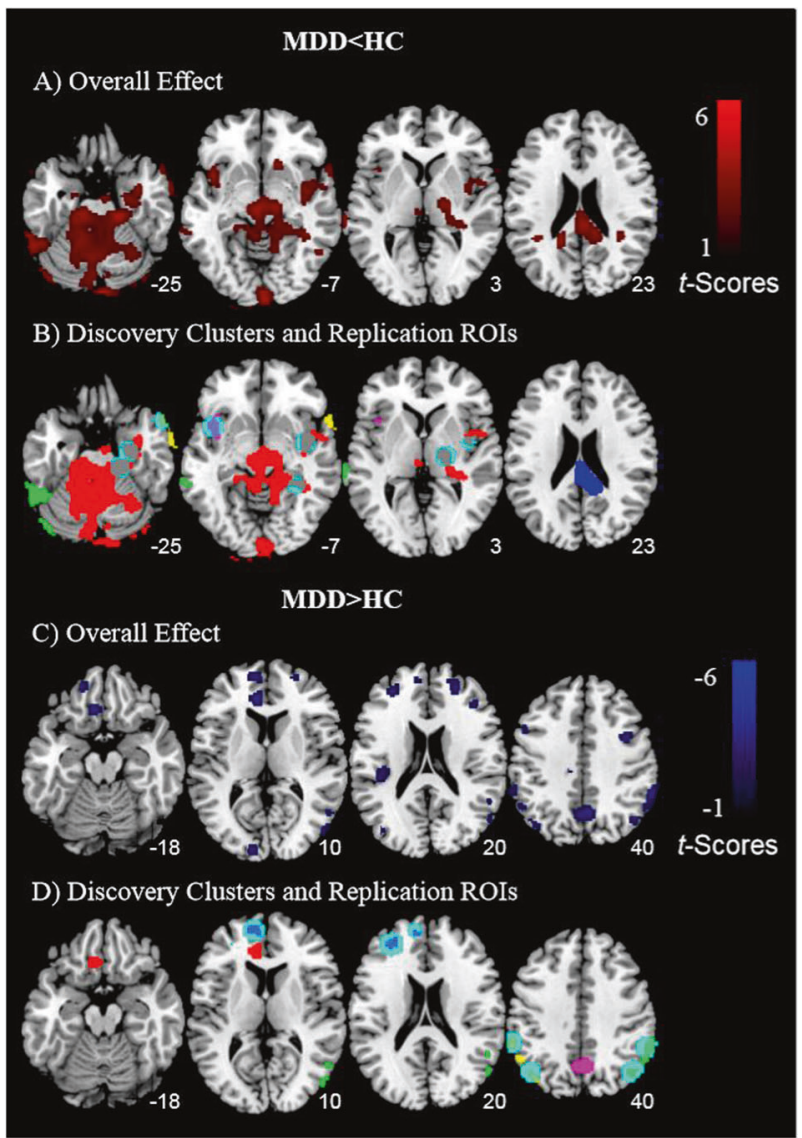

Fig. 3 Brain regions in which groups showed differences in relative cerebral blood flow (rCBF; perfusion). a Overall effect of Group controlling for Site where participants with major depressive disorder (MDD) had lower rCBF relative to healthy controls (HC). b Regions for which $\mathrm{rCBF}$ was lower for those with MDD relative to HC. Discovery clusters are represented in color based on Table 1 definitions (Cluster 1-Red; Cluster 2-Blue; Cluster 3-Green; Cluster 4-Yellow; and Cluster 5-Violet) and the Replication region of interest-spheres (ROIs), derived from the Discovery clusters, imposed in cyan. c Overall effect of Group controlling for Site where MDD participants had greater $\mathrm{rCBF}$ relative to HC. d Regions for which rCBF for was greater in those with MDD than HC. Discovery clusters are represented in color based on Table 1 definitions (Cluster 1-Red; Cluster 2-Blue; Cluster 3-Green; Cluster 4-Yellow; and Cluster 5-Violet) and replication ROIs imposed in cyan

in the MDD group relative to $\mathrm{HC}$, only $\mathrm{rCBF}$ in the supramarginal gyrus was negatively correlated with duration of current episode, such that longer episode duration was associated with lower perfusion $\left(r_{\mathrm{s}}=-0.26 ; p<0.05\right)$.

\section{Discussion}

The present study is the first to discover and replicate $\mathrm{rCBF}$ differences in MDD using a large, well characterized population. The study examined 164 MDD participants and divided them into 2/3-1/3 Discovery-Replication samples.
The Discovery sample provided further evidence for the role of ASL in detecting CBF differences in brain regions implicated in the phenotype of MDD [17-19]. The Replication sample provided independent, unbiased confirmation for these rCBF differences. Both samples revealed that $\mathrm{HC}$ have consistently increased perfusion relative to MDD participants, whose dysfunction in these regions has been characterized using other MRI modalities examining brain networks. Specifically, the midbrain cluster encompassed regions involved in multiple cognitive functions, sleep, salience, and reward processing, the insula in salience, interoceptive and emotion processing, and regions of the temporal lobe in higher order sensory processing [20-27]. HC participants had a majority of their perfusion differences in the frontal lobe (executive function), while MDD participants' were in the inferior parietal lobule (angular gyrus; default-mode network and attention) highlighting shifts in cognitive networks [28, 29]. Disruptions in connectivity within, and between, these large networks arguably play a significant role in the pathophysiology of MDD such as persistent negative mood, emotion processing, self-focus, and ruminating thoughts [30-32].

In addition to observing replicable lower perfusion, this study's rCBF findings also revealed MDD participants to have replicable greater perfusion than $\mathrm{HC}$ in other brain regions. Nearly all ASL research in MDD has only observed $\mathrm{CBF}$ to be lower in those with MDD relative to $\mathrm{HC}$, and not greater rCBF or both $[5,7,8]$. However, one other study that scanned treatment-responders, prior to starting their antidepressant treatment, observed greater CBF in MDD than HC in regions of the occipital lobe and limbic system [8]. The MDD samples used for the EMBARC Discovery-Replication analyses likely include responders and non-responders to antidepressant treatment, limiting replication. The heterogeneity of the disease within this population may also limit consistency across all findings. Other considerations are their complicated symptom profile and the sensitivity of the CBF measure implemented (aCBF vs. rCBF).

The replication of $52 \%$ of our discovery-derived clusters is exciting given the lack of discovery-replication work in the field. This is augmented by the associations detected between these replicated clusters and MDD clinical features. The majority of the clinical-perfusion relationships were chronicity-based MDD features. Associations were detected for rCBF and duration of current episode (parahippocampal and supramarginal gyri), length of illness (thalamus and insula), age of onset (insula), and number of MDEs (thalamus). These chronicity-based relationships may suggest that levels of perfusion in MDD could be markers of trait or longterm effects of MDD (i.e., brain scarring). Paired with the observation that most of these relationships existed for regions that were also identified to have medium to large effect sizes between MDD and HC, these regions may not just provide 
markers of disease, but may also provide mediators of treatment response.

\section{Limitations}

To reduce biologic heterogeneity, EMBARC participants had chronic, early-onset MDD. This may limit the generalizability of the findings to newly depressed or latestage MDD populations. The DLBS study did not collect clinically relevant information like EMBARC. The inability to confirm that the DLBS HC sample clinically matched the EMBARC HC sample is a limitation and may play a role in some replication discrepancies. Other effects such as age or MDD severity may not have been completely controlled for in the pseudo-randomization of the MDD population into the Discovery and Replication samples. rCBF did not differ by gender in the MDD sample, but thalamic rCBF was positively correlated with age prior to multiple comparison correction. Residual effects of age on rCBF could be of interest with studies of a wider range and greater representation by age. EMBARC's protocols optimized quality and performance while maintaining similar parameters (in line with ASL recommendations $[2,33])$ and revealed great $\mathrm{HC}$ test-retest reliability [12]. However, site variations could also still play a role in Discovery-Replication discrepancies despite use of $\mathrm{rCBF}$. The inclusion of the DLBS HC likely impacted the ability to adequately control for site variations in the Replication analyses. Future methodological work on across- and within-site scanner reliability and between-group effects could still assist in understanding CBF data and its discrepancies in MDD. ASL data compared to other imaging modalities can have a lower signal-to-noise ratio and magnetic susceptibility signal voiding/artifact in regions like the frontal lobes. This could play an additional role in the inconsistent findings of the prefrontal regions. A recent study investigating $\mathrm{CBF}$ and glucose metabolism in MDD observed glucose metabolism to be more sensitive than CBF in identifying functional abnormalities in the frontal lobes [34]. Perfusion, neuronal activity, and metabolism of a given region may inform or influence each other. Recent findings on $\mathrm{CBF}$ and cerebral metabolic rate of oxygen identified a 2:1 ratio of perfusion to neuronal consumption that mediated the BOLD signature in aging populations, suggesting age-related changes in perfusion and neuronal activity are not equal, and mediate one another [35]. MDD-related changes in these brain physiology relationships might also exist to some degree. Modeling other imaging measures with $\mathrm{rCBF}$ may assist in better characterization of perfusion markers in MDD.

\section{Future directions}

ASL has become a more widely used research tool, but of the many MRI modalities, it has ideal prospects of being translated into a tool for clinical diagnostics and informing treatment decisions. The short scan-time, capacity for automated processing, and direct, quantifiable physiological measure are all desired qualities of a biomarker. In combination with clinical symptom profiles, CBF-defined regional disruptions could inform tailored antidepressant or therapy programs, or even provide anatomical targets for treatments (e.g., transcranial magnetic stimulation). Beyond its utility in diagnosing and choosing treatment, CBF can provide a noninvasive biomarker of disease progression and possibly predict relapse by tracking changes, or lack thereof, in perfusion over time.

The current study is the first to present independent internal and external replication of $\mathrm{CBF}$ in MDD for regions that lack and exceed perfusions levels of $\mathrm{HC}$. The replication and overlap in brain regions detected in our perfusion data and those investigated using other imaging modalities of treatment response is promising for the future development of reliable MDD biosignatures (e.g., default-mode network connectivity and serotonin norepinephrine reuptake inhibitor treatment [36]; insula metabolism and cognitive behavioral therapy vs selective serotonin reuptake inhibitors [37]; superior temporal gyrus and insula gray matter volume and transcranial magnetic stimulation [38]; for a review of resting fMRI and various treatments, see [39]). These ASL findings have implications for future studies aimed to develop CBF as both a diagnostic and treatment biomarker in clinical populations. A follow-up study using the complete EMBARC outcomes dataset with 300 MDD participants may provide further information regarding the application of $\mathrm{CBF}$ in predicting treatment response in MDD.

Acknowledgements The EMBARC study reported in this publication was supported by the National Institute of Mental Health of the National Institutes of Health under award numbers U01MH092221 (Trivedi MH PI) and U01MH092250 (Weissman MM PI). The content is solely the responsibility of the authors and does not necessarily represent the official views of the National Institutes of Health. This work was supported by the EMBARC National Coordinating Center at UT Southwestern Medical Center and Data Center at Columbia University. Thomas S. Harris provided assistance with ASL data processing and analytic design. We thank Jennifer Furman, Ph.D. for her administrative assistance. The healthy control data, used to supplement analyses, were collected as part of the Dallas Lifespan Brain Study (http://fcon_1000.projects.nitrc.org/ indi/retro/dlbs.html), funded by R37 AG006265 (Study PI: Denise Park), R21 AG034318, and R01 MH084021 (ASL PI: Hanzhang $\mathrm{Lu}$ ). Dr. Hanzhang Lu served as one of the EMBARC MR Physicists and the ASL consultant. Bruce Grannemann died on 9 January, 2019. We wish to dedicate this publication to him and the significant role he played in this work. 


\section{Compliance with ethical standards}

Conflict of interest Crystal Cooper, Cherise Chin Fatt, Peiying Liu, Bruce Grannemann, Thomas Carmody, Jorge Almeida, Ashley Malchow, Melvin McInnis, Ramin Parsey, Elizabeth Bartlett, and Hanzhang $\mathrm{Lu}$ declare that they have no conflict of interest. Thilo Deckersbach's research has been funded by NIH, NIMH, NARSAD, TSA, IOCDF, Tufts University, DBDAT, Otsuka Pharmaceuticals, Cogito, Inc. and Sunovian. He has received honoraria, consultation fees and/or royalties from the MGH Psychiatry Academy, BrainCells Inc., Clintara, LLC., Systems Research and Applications Corporation, Boston University, the Catalan Agency for Health Technology Assessment and Research, the National Association of Social Workers Massachusetts, the Massachusetts Medical Society, Tufts University, NIDA, NIMH, and Oxford University Press. He has also participated in research funded by DARPA, NIH, NIMH, NIA, AHRQ, PCORI, Janssen Pharmaceuticals, The Forest Research Institute, Shire Development Inc., Medtronic, Cyberonics, Northstar, Takeda and Alphasigma. Maurizio Fava has received research support from Abbott Laboratories; Acadia Pharmaceuticals; Alkermes, Inc.; American Cyanamid;Aspect Medical Systems; AstraZeneca; Avanir Pharmaceuticals; AXSOME Therapeutics; BioResearch; BrainCells Inc.; BristolMyers Squibb; CeNeRx BioPharma; Cephalon; Cerecor; Clintara, LLC; Covance; Covidien; Eli Lilly and Company;EnVivo Pharmaceuticals, Inc.; Euthymics Bioscience, Inc.; Forest Pharmaceuticals, Inc.; FORUM Pharmaceuticals; Ganeden Biotech, Inc.; GlaxoSmithKline; Harvard Clinical Research Institute; Hoffman-LaRoche; Icon Clinical Research; i3 Innovus/Ingenix; Janssen R\&D, LLC; Jed Foundation; Johnson \& Johnson Pharmaceutical Research \& Development; Lichtwer Pharma GmbH; Lorex Pharmaceuticals; Lundbeck Inc.; MedAvante; Methylation Sciences Inc; National Alliance for Research on Schizophrenia \& Depression (NARSAD); National Center for Complementary and Alternative Medicine (NCCAM); National Coordinating Center for Integrated Medicine (NiiCM); National Institute of Drug Abuse (NIDA); National Institute of Mental Health (NIMH); Neuralstem, Inc.; NeuroRx; Novartis AG; Organon Pharmaceuticals; PamLab, LLC.; Pfizer Inc.; Pharmacia-Upjohn; Pharmaceutical Research Associates., Inc.; Pharmavite ${ }^{\circledR}$ LLC;PharmoRx Therapeutics; Photothera; Reckitt Benckiser; Roche Pharmaceuticals; RCT Logic, LLC (formerly Clinical Trials Solutions, LLC); SanofiAventis US LLC; Shire; Solvay Pharmaceuticals, Inc.; Stanley Medical Research Institute (SMRI); Synthelabo; Takeda Pharmaceuticals;Tal Medical; VistaGen); Wyeth-Ayerst Laboratories; he has served as advisor or consultant to Abbott Laboratories; Acadia; Affectis Pharmaceuticals AG; Alkermes, Inc.; Amarin Pharma Inc.; Aspect Medical Systems; AstraZeneca; Auspex Pharmaceuticals; Avanir Pharmaceuticals; AXSOME Therapeutics; Bayer AG; Best Practice Project Management, Inc.; Biogen; BioMarin Pharmaceuticals, Inc.; Biovail Corporation; BrainCells Inc; Bristol-Myers Squibb; CeNeRx BioPharma; Cephalon, Inc.; Cerecor; CNS Response, Inc.; Compellis Pharmaceuticals; Cypress Pharmaceutical, Inc.; DiagnoSearch Life Sciences (P) Ltd.; Dinippon Sumitomo Pharma Co. Inc.; Dov Pharmaceuticals, Inc.; Edgemont Pharmaceuticals, Inc.; Eisai Inc.; Eli Lilly and Company; EnVivo Pharmaceuticals, Inc.; ePharmaSolutions; EPIX Pharmaceuticals, Inc.; Euthymics Bioscience, Inc.; Fabre-Kramer Pharmaceuticals, Inc.; Forest Pharmaceuticals, Inc.; Forum Pharmaceuticals; GenOmind, LLC; GlaxoSmithKline; Grunenthal GmbH; Indivior; i3 Innovus/Ingenis; Intracellular; Janssen Pharmaceutica; Jazz Pharmaceuticals, Inc.; Johnson \& Johnson Pharmaceutical Research \& Development, LLC; Knoll Pharmaceuticals Corp.; Labopharm Inc.; Lorex Pharmaceuticals; Lundbeck Inc.; MedAvante, Inc.; Merck \& Co., Inc.; MSI Methylation Sciences, Inc.; Naurex, Inc.; Nestle Health Sciences; Neuralstem, Inc.; Neuronetics, Inc.; NextWave Pharmaceuticals; Novartis AG; Nutrition 21; Orexigen Therapeutics, Inc.; Organon Pharmaceuticals; Osmotica; Otsuka Pharmaceuticals; Pamlab,
LLC.; Pfizer Inc.; PharmaStar; Pharmavite ${ }^{\circledR}$ LLC.; PharmoRx Therapeutics; Precision Human Biolaboratory; Prexa Pharmaceuticals, Inc.; PPD; Puretech Ventures; PsychoGenics; Psylin Neurosciences, Inc.; RCT Logic, LLC (formerly Clinical Trials Solutions, LLC); Rexahn Pharmaceuticals, Inc.; Ridge Diagnostics, Inc.; Roche; Sanofi-Aventis US LLC.; Sepracor Inc.; Servier Laboratories; Schering-Plough Corporation; Shenox Pharmaceuticals; Solvay Pharmaceuticals, Inc.; Somaxon Pharmaceuticals, Inc.; Somerset Pharmaceuticals, Inc.; Sunovion Pharmaceuticals; Supernus Pharmaceuticals, Inc.; Synthelabo; Taisho Pharmaceutical; Takeda Pharmaceutical Company Limited; Tal Medical, Inc.; Tetragenex Pharmaceuticals, Inc.; TransForm Pharmaceuticals, Inc.; Transcept Pharmaceuticals, Inc.; Vanda Pharmaceuticals, Inc.; VistaGen; he has received speaking or publishing fees from Adamed, Co; Advanced Meeting Partners; American Psychiatric Association; American Society of Clinical Psychopharmacology; AstraZeneca; Belvoir Media Group; Boehringer Ingelheim GmbH; Bristol-Myers Squibb; Cephalon, Inc.; CME Institute/Physicians Postgraduate Press, Inc.; Eli Lilly and Company; Forest Pharmaceuticals, Inc.; GlaxoSmithKline; Imedex, LLC; MGH Psychiatry Academy/Primedia; MGH Psychiatry Academy/Reed Elsevier; Novartis AG; Organon Pharmaceuticals; Pfizer Inc.; PharmaStar; United BioSource,Corp.; Wyeth-Ayerst Laboratories; he has equity holdings in Compellis and PsyBrain, Inc.; he has a patent for Sequential Parallel Comparison Design (SPCD), which are licensed by MGH to Pharmaceutical Product Development, LLC (PPD); and patent application for a combination of Ketamine plus Scopolamine in Major Depressive Disorder (MDD), licensed by MGH to Biohaven; and he receives copyright royalties for the MGH Cognitive \& Physical Functioning Questionnaire (CPFQ), Sexual Functioning Inventory (SFI), Antidepressant Treatment Response Questionnaire (ATRQ), Discontinuation-Emergent Signs \& Symptoms (DESS), Symptoms of Depression Questionnaire (SDQ), and SAFER; Lippincott, Williams \& Wilkins; Wolkers Kluwer; World Scientific Publishing Co. Pte. Ltd. Benji Kurian has received grant support from Targacept, Inc., Pfizer, Inc., Johnson \& Johnson, Evotec, Rexahn, Naurex, Forest Pharmaceuticals, and NIMH. Patrick McGrath has received funding from the National Institute of Mental Health, New York State Department of Mental Hygiene, Research Foundation for Mental Hygiene (New York State), Forest Research Laboratories, Sunovion Pharmaceuticals, and Naurex Pharmaceuticals (now Allergan). Maria Oquendo receives royalties for use of the Columbia Suicide Severity Rating Scale. Her family owns stock in Bristol-Myers Squibb. Myrna Weissman received funding from the National Institute of Mental Health (NIMH), the National Institute on Drug Abuse. (NIDA), the National Alliance for Research on Schizophrenia and Depression. (NARSAD), the Sackler Foundation, and the Templeton Foundation; and receives royalties from the Oxford University Press, Perseus Press, the American Psychiatric Association Press, and MultiHealth Systems. Mary Phillips received funding from the National Institute of Mental Health (NIMH). Madhukar Trivedi is or has been an advisor/consultant and received fee from (lifetime disclosure): Abbott Laboratories Inc., Akzo (Organon Pharmaceuticals Inc.), Allergan Sales LLC, Alkermes, Arcadia Pharmaceuticals Inc., AstraZeneca, Axon Advisors, Brintellix, BristolMyers Squibb Company, Cephalon Inc., Cerecor, Eli Lilly \& Company, Evotec, Fabre-Kramer Pharmaceuticals Inc., Forest Pharmaceuticals, GlaxoSmithKline, Global Medical Education Inc., Health Research Associates, Johnson \& Johnson, Lundbeck, MedAvante Medscape, Medtronic, Merck, Mitsubishi Tanabe Pharma Development America Inc., MSI Methylation Sciences Inc., Nestle Health Science-PamLab Inc., Naurex, Neuronetics, One Carbon Therapeutics Ltd., Otsuka Pharmaceuticals, Pamlab, Parke-Davis Pharmaceuticals Inc., Pfizer Inc., PgxHealth, Phoenix Marketing Solutions, Rexahn Pharmaceuticals, Ridge Diagnostics, Roche Products Ltd., Sepracor, SHIRE Development, Sierra, SK Life and Science, Sunovion, Takeda, Tal Medical/Puretech Venture, Targacept, Transcept, VantagePoint, 
Vivus, and Wyeth-Ayerst Laboratories. In addition, he has received grants/research support from: Agency for Healthcare Research and Quality, Cyberonics Inc., National Alliance for Research in Schizophrenia and Depression, National Institute of Mental Health, National Institute on Drug Abuse, National Institute of Diabetes and Digestive and Kidney Diseases, Johnson \& Johnson; and he receives royalties from Janssen Research and Development LLC.

Publisher's note: Springer Nature remains neutral with regard to jurisdictional claims in published maps and institutional affiliations.

\section{References}

1. Phillips ML, Chase HW, Sheline YI, Etkin A, Almeida JR, Deckersbach $\mathrm{T}$, et al. Identifying predictors, moderators, and mediators of antidepressant response in major depressive disorder: neuroimaging approaches. Am J Psychiatry. 2015;172:124-38.

2. Alsop DC, Detre JA, Golay X, Günther M, Hendrikse J, HernandezGarcia L, et al. Recommended implementation of arterial spinlabeled perfusion MRI for clinical applications: a consensus of the ISMRM perfusion study group and the European consortium for ASL in dementia. Magn Reson Med. 2015;73:102-16.

3. Orosz A, Jann K, Federspiel A, Horn H, Höfle O, Dierks T, et al. Reduced cerebral blood flow within the default-mode network and within total gray matter in major depression. Brain Connect. 2012;2:303-10.

4. Almeida JRC, Mourao-Miranda J, Aizenstein HJ, Versace A, Kozel FA, Lu H, et al. Pattern recognition analysis of anterior cingulate cortex blood flow to classify bipolar v. unipolar depression. Br J Psychiatry. 2013;203:310-1.

5. Ota M, Noda T, Sato N, Hattori K, Teraishi T, Hori H, et al. Characteristic distributions of regional cerebral blood flow changes in major depressive disorder patients: a pseudocontinuous arterial spin labeling (pCASL) study. J Affect Disord. 2014;165:59-63.

6. Trivedi MH, McGrath PJ, Fava M, Parsey RV, Kurian BT, Phillips ML, et al. Establishing moderators and biosignatures of antidepressant response in clinical care (EMBARC): rationale and design. J Psychiatr Res. 2016;78:11-23.

7. Järnum H, Eskildsen SF, Steffensen EG, Lundbye-Christensen S, Simonsen CW, Thomsen IS, et al. Longitudinal MRI study of cortical thickness, perfusion, and metabolite levels in major depressive disorder. Acta Psychiatr Scand. 2011;124:435-46.

8. Lui S, Parkes LM, Huang X, Zou K, Chan RC, Yang H, et al. Depressive disorders: focally altered cerebral perfusion measured with arterial spin-labeling MR imaging. Radiology. 2009;251:476-84.

9. van den Oord EJ, Kuo PH, Hartmann AM, Webb BT, Möller HJ, Hettema JM, et al. Genomewide association analysis followed by a replication study implicates a novel candidate gene for neuroticism. Arch Gen Psychiatry. 2008;65:1062-71.

10. First MB, Spitzer RL, Gibbon M, Williams JW. Structured Clinical Interview for DSM-IV-TR Axis I Disorders, Research Version, Patient Edition With Psychotic Screen (SCID-I/P W/ PSY SCREEN). New York: Biometrics Research, New York State Psychiatric Institute; 2002.

11. Rush AJ, Trivedi MH, Ibrahim HM, Carmody TJ, Arnow B, Klein $\mathrm{DN}$, et al. The 16-item quick inventory of depressive symptomatology (QIDS), clinician rating (QIDS-C), and self-report (QIDS-SR): a psychometric evaluation in patients with chronic major depression. Biol Psychiatry. 2003;54:573-83.

12. Almeida JRC, Greenberg T, Lu H, Chase HW, Fournier J, Cooper $\mathrm{CM}$, et al. Test-retest reliability of cerebral blood flow in healthy individuals using arterial spin labeling: findings from the EMBARC study. Magn Reson Imaging. 2017;45:26-33.

13. Jann K, Gee DG, Kilroy E, Schwab S, Smith RX, Cannon TD, et al. Functional connectivity in BOLD and CBF data: similarity and reliability of resting brain networks. Neuroimage. 2015; 106:111-22.

14. Aslan S, Lu H. On the sensitivity of ASL MRI in detecting regional differences in cerebral blood flow. Magn Reson Imaging. 2010;28:928-35.

15. Tzourio-Mazoyer N, Landeau B, Papathanassiou D, Crivello F, Etard O, Delcroix N, et al. Automated anatomical labeling of activations in SPM using a macroscopic anatomical parcellation of the MNI MRI single-subject brain. Neuroimage. 2002;15:273-89.

16. Hamilton M. A rating scale for depression. J Neurol Neurosurg Psychiatry. 1960;23:56-62.

17. Crossley NA, Mechelli A, Scott J, Carletti F, Fox PT, McGuire P, et al. The hubs of the human connectome are generally implicated in the anatomy of brain disorders. Brain. 2014;137:2382-95.

18. Disner SG, Beevers CG, Haigh EA, Beck AT. Neural mechanisms of the cognitive model of depression. Nat Rev Neurosci. 2011;12:467-77.

19. Kaiser RH, Andrews-Hanna JR, Wager TD, Pizzagalli DA. Largescale network dysfunction in major depressive disorder: metaanalysis of resting-state functional connectivity. JAMA Psychiatry. 2015;72:603-11.

20. Andrews-Hanna JR, Smallwood J, Spreng RN. The default network and self-generated thought: component processes, dynamic control, and clinical relevance. Ann N Y Acad Sci. 2014;1316:29-52.

21. Fornito A, Zalesky A, Breakspear M. The connectomics of brain disorders. Nat Rev Neurosci. 2015;16:159-72.

22. Hamilton JP, Furman DJ, Chang C, Thomason ME, Dennis E, Gotlib IH. Default-mode and task-positive network activity in major depressive disorder: implications for adaptive and maladaptive rumination. Biol Psychiatry. 2011;70:327-33.

23. Menon V. Salience network. Brain Mapp: Encycl Ref. 2015; 2:597-611.

24. Menon V, Uddin LQ. Saliency, switching, attention and control: a network model of insula function. Brain Struct Funct. 2010; 214:655-67.

25. Seeley WW, Menon V, Schatzberg AF, Keller J, Glover GH, Kenna $\mathrm{H}$, et al. Dissociable intrinsic connectivity networks for salience processing and executive control. J Neurosci. 2007;27:2349-56.

26. Wang L, Li X, Li K, Su Y, Zeng Y, Zhang Q, et al. Mapping the effect of escitalopram treatment on amplitude of low-frequency fluctuations in patients with depression: a resting-state fMRI study. Metab Brain Dis. 2017;32:147-54.

27. Young CB, Chen T, Nusslock R, Keller J, Schatzberg AF, Menon $\mathrm{V}$. Anhedonia and general distress show dissociable ventromedial prefrontal cortex connectivity in major depressive disorder. Transl Psychiatry. 2016;6:e810.

28. Vatansever D, Manktelow AE, Sahakian BJ, Menon DK, Stamatakis EA. Angular default mode network connectivity across working memory load. Hum Brain Mapp. 2017;38:41-52.

29. Bressler SL, Menon V. Large-scale brain networks in cognition: emerging methods and principles. Trends Cogn Sci. 2010;14:277-90.

30. Bassett DS, Bullmore ET. Human brain networks in health and disease. Curr Opin Neurol. 2009;22:340-7.

31. Menon V. Large-scale brain networks and psychopathology: a unifying triple network model. Trends Cogn Sci. 2011;15:483-506.

32. Singh MK, Kesler SR, Hadi Hosseini SM, Kelley RG, Amatya D, Hamilton JP, et al. Anomalous gray matter structural networks in major depressive disorder. Biol Psychiatry. 2013;74:777-85. 
33. Mutsaerts HJ, van Osch MJ, Zelaya FO, Wang DJ, Nordhøy W, Wang Y, et al. Multi-vendor reliability of arterial spin labeling perfusion MRI using a near-identical sequence: implications for multi-center studies. Neuroimage. 2015;113:143-52.

34. Fu C, Shi D, Gao Y, Xu J. Functional assessment of prefrontal lobes in patients with major depression disorder using a dualmode technique of 3D-arterial spin labeling and $18 \mathrm{~F}$ fluorodeoxyglucose positron emission tomography/computed tomography. Exp Ther Med. 2017;14:1058-64.

35. Hutchison JL, Lu H, Rypma B. Neural mechanisms of age-related slowing: the $\triangle \mathrm{CBF} / \triangle \mathrm{CMRO} 2$ ratio mediates age-differences in BOLD signal and human performance. Cereb Cortex. 2013; 23:2337-46.

36. Fu CH, Costafreda SG, Sankar A, Adams TM, Rasenick MM, Liu $\mathrm{P}$, et al. Multimodal functional and structural neuroimaging investigation of major depressive disorder following treatment with duloxetine. BMC Psychiatry. 2015;15:82.

37. McGrath CL, Kelley ME, Holtzheimer PE, Dunlop BW, Craighead WE, Franco AR, et al. Toward a neuroimaging treatment selection biomarker for major depressive disorder. JAMA Psychiatry. 2013;70:821-9.

38. Lan MJ, Chhetry BT, Liston C, Mann JJ, Dubin M. Transcranial magnetic stimulation of left dorsolateral prefrontal cortex induces brain morphological changes in regions associated with a treatment resistant major depressive episode: an exploratory analysis. Brain Stimul. 2016;9:577-83.

39. Dichter GS, Gibbs D, Smoski MJ. A systematic review of relations between resting-state functional-MRI and treatment response in major depressive disorder. J Affect Disord. 2015; 172:8-17. 